\title{
Difficulties in the treatment of acute pulmonary embolism
}

Although acute pulmonary embolism is a common condition many clinicians have serious doubts about the correct treatment for individual patients. The reason for these doubts is simple; there is a lack of conclusive clinical data on which to base decisions. There are several important questions for the clinician when faced with such patients: (1) Does the patient need thrombolytic treatment? (2) How should heparin be administered? (3) Should venography with a view to venous interruption be performed routinely in patients who have suffered definite pulmonary embolism?

\section{Is thrombolytic treatment needed?}

Thrombolytic treatment is not needed in patients who have suffered minor acute pulmonary embolism or even massive pulmonary embolism (defined angiographically as greater than $50 \%$ obstruction of the pulmonary arterial bed) unless there is considerable haemodynamic embarrassment. These patients do well with heparin, which drastically reduces the risk of further embolism, while natural lytic processes remove thrombus from the pulmonary and venous circulations..$^{1-3}$ Thrombolytic treatment does not reduce early mortality ${ }^{2}$ or improve long term clinical results. ${ }^{4}$ Subtle abnormalities of pulmonary function due to capillary damage are much less one year after the event in patients who have received thrombolytic treatment, but the clinical significance of these findings is unknown $n^{5}$ and probably unimportant.

Randomised trials comparing heparin and thrombolytic treatment have shown conclusively that emboli clear much more rapidly from pulmonary arteries in those patients receiving thrombolytic drugs $^{23}$ but in these trials there was no difference in mortality. Although, theoretically, a benefit might be expected as a result of more rapid clearing of the clot from the pulmonary arteries, thrombolytic treatment has never been shown to affect mortality. This may be because no benefit exists or because the design of these trials could not be expected to produce an answer. The latter explanation is probably

Address for reprint requests: Dr Roger Hall, Royal Victoria Infirmary, Newcastle Upon Tyne NE1 4LP. correct. Since the early death rate is high, survival in acute massive pulmonary embolism is a function of the time that elapses after the embolism before the patient is first seen. ${ }^{6}$ By the time referral to a centre, enrolment into the trial, and investigations such as angiography have taken place a group of "survivors" has already been selected.

It is unlikely that an adequate comparison of heparin with thrombolytic treatment, restricted to haemodynamically compromised patients seen early after embolism, will ever be performed. Firstly, the logistic problem of collecting similar groups of patients are enormous. Secondly, the knowledge that thrombolytic treatment can clear thrombus rapidly from the pulmonary arteries makes it impossible to withhold it from the patient deteriorating with heparin treatment even though, because of the way the clinical condition can alter rapidly in the early stage after embolism, some of these deteriorating patients might be expected to survive eventually without any change in treatment. The incidence of "treatment failure" (defined as clinical deterioration despite adequate treatment requiring escalation of treatment to thrombolytic medication or embolectomy) was much higher in patients receiving heparin for life threatening massive pulmonary embolism $(30 \%)$ than in patients receiving thrombolytic treatment $(15 \%))^{7}$ Treatment failure occurred exclusively among the patients with the most severe haemodynamic disturbance and a systolic blood pressure of $100 \mathrm{~mm} \mathrm{Hg}$ or less when first seen. A similar pattern of treatment failure was also reported by Miller et al. ${ }^{8}$ Although considerably more expensive than heparin, thrombolytic medication has few other disadvantages by comparison with heparin. Both agents are dangerous if there is a bleeding tendency and thrombolytic treatment is probably particularly dangerous in this respect within the first week after major surgery or trauma. The risk of serious haemorrhage is similar (4\% for heparin and $9 \%$ for thrombolytic drugs in the urokinase study ${ }^{2}$ and $7 \%$ in both groups in an earlier study). ${ }^{3}$

Since there are possible advantages without a serious increase in disadvantages, thrombolytic treatment should be used in the haemodynamically compromised patient. Useful guidelines for identifying such patients are sustained hypotension, 
peripheral circulatory shutdown, poor urine flow, and severe hypoxia. Twelve to 24 hours of treatment are usually adequate.

Once the clinician has decided that the patient fits this picture the next dilemma is whether the patient should undergo a pulmonary angiogram before thrombolytic treatment is begun. Although some authors $^{y 10}$ regard this as mandatory, their view ignores the clinical realities. Most massive pulmonary emboli occur in hospitals that do not have facilities for pulmonary angiography. Transfer of such critically ill patients to a suitable centre is usually not appropriate because of the time required and the rigors of the journey. Attempted emergency angiography in the radiography departments of most district general hospitals is likely to be dangerous and unhelpful. Although errors in the diagnosis of pulmonary embolism are common, this is more frequent with smaller emboli and many patients with large emboli are encountered in whom on clinical grounds, possibly with the help of perfusion lung scanning, diagnostic certainty is sufficiently strong to allow thrombolytic treatment without prior angiography. If there is any doubt and an alternative cause for collapse cannot be found, transfer to a specialist centre may have to be undertaken.

As soon as a diagnosis of pulmonary embolism is strongly suspected and while these difficult decisions are being taken, intravenous heparin should be given as a bolus (10 $000 \mathrm{IU}$ for the average sized adult).

\section{How should heparin be administered?}

Heparin has been available for clinical use for nearly 50 years but many physicians still have considerable doubts about the correct way to use it. The object of heparin treatment is simple: to give enough to prevent recurrent thrombosis and embolism while avoiding haemorrhagic complications. The problems arise from a lack of conclusive evidence about the best and safest method of administration, whether dosage should be arbitrary or controlled by clotting tests to achieve maximum safety combined with effectiveness, and how long the drug should be given for. These would, at first sight, seem easy questions to answer but the extent of published work on heparin treatment proves that this is not so. Studies have often been far from ideal, containing patients with a wide variety of thromboembolic disorders sometimes mixed with patients simply receiving prophylactic anticoagulation. ${ }^{11}$ The rate at which heparin is consumed varies with the degree of thrombosis and tends to be higher when this is extensive. ${ }^{12}$ Furthermore, heparin's anticoagulant activity varies with the patient's own serum level of antithrombin III activity, which can be altered by a congenital metabolic defect, thrombosis itself, and the administration of heparin. If the antithrombin III activity is reduced below $60 \%$ of normal the effectiveness of heparin as an anticoagulant may be severely impaired. ${ }^{13}$ Anticoagulation levels deemed to be effective have been often arbitrarily predetermined by extrapolation from animal studies, and groups receiving heparin by different techniques (infusion or bolus injection) have often received different overall doses of heparin. ${ }^{114-17}$ The issue is further confused by the recent discovery of wide diurnal variation in the results of clotting tests (activated prothrombin time, thrombin time, and assay of factor $\mathrm{Xa}$ ) in patients receiving heparin, the lowest activity occurring around 8 am and the highest around midnight. ${ }^{18}$ Since most studies have not standardised the time of day at which tests used to control heparin dosage are performed, their results must be regarded with caution. The finding of diurnal fluctuations in heparin activity of as much as $50 \%$ may explain why most studies have shown that, despite careful control of heparin dosage, only about half the measurements fall in the desired therapeutic range. ${ }^{141517}$ It may also explain why all but a few studies have failed to show a correlation between the results of clotting tests and the incidence of either further thromboembolism or serious haemorrhage.

The intricacy of these problems is well summarised by the excellent but extremely complicated study of Wilson et al. ${ }^{17}$ Like others before them, they suggest that the main determinant of recurrence and haemorrhagic complications is the dose of heparin administered. 1114 is In general, studies comparing intermittent (usually four hourly) injection of heparin and continuous infusion have shown that haemorrhagic complications are lower in patients having the latter, who also tend to receive lower overall dosages. ${ }^{11} 1516$ The only study which found a higher incidence of complications with infusion is also the only one in which this group received the higher dosage. ${ }^{14}$ Patients receiving a continuous infusion receive a lower dose for various reasons. In some studies this is because intermittent dosage is arbitrary while continuous dosage is adjusted according to the results of clotting tests, while in others the intermittent dosage is tailored to give an anticoagulant effect similar to that of continuous treatment just before the next dose. This needs high doses because of the rapid clearance of heparin from the circulation after the bolus. Most studies have shown that further embolism is reduced to a similar extent by the two methods, but these studies have often contained too few patients at high risk of further pulmonary embolism to permit valid conclu- 
sions to be drawn. Wilson et al, ${ }^{17}$ who studied a large group of patients, all of whom had thromboembolic disease, found the recurrence rate to be higher in patients receiving a continuous infusion (and as usual less heparin than the intermittent injection group). They also showed that haemorrhagic complications with intermittent injection occurred more frequently when there was a complicating risk factor; the most important factors were age (over 60 years), uraemia, a pre-existing haemostatic defect, severe hypertension, recent surgery, previous gastrointestinal haemorrhage, or major pulmonary embolism. They also found that patients who bled had, as a group, received more heparin than those who did not. The risk factors, however, seem to have been retrospectively determined and, since mortality was similar whether intermittent or continuous anticoagulation was used, it seems that those receiving intermittent heparin have a small but definite risk of dying of haemorrhage, whereas those receiving a continuous infusion have a similar risk of death but in their case from recurrent embolism. Despite the enormous amount of work done in this careful study it is still unknown whether increasing the dose given by continuous infusion to the level of the intermittent doses would have reduced recurrence without increasing haemorrhage or whether a corresponding reduction in the intermittent dose would have reduced haemorrhage without increasing recurrence.

One area of unanimity is the choice of clotting test. There seems little to choose between them and the most commonly used are activated partial thromboplastin time, thrombin time, and Lee-White clotting time. The aim is usually to maintain a plasma heparin level of $0.3-0.4$ units $/ \mathrm{ml}$, a figure judged from animal experiments to be effective in preventing clot extension. This requires the first two tests to have a value from one and a half times to twice the control value and the last to be between two and three times the control value.

Although there are strong protagonists for infusion and for intermittent injection as well as for the routine use of frequent clotting tests to control dosage, the only fair conclusion is that there is little or no difference between the two techniques and that frequent tinkering with dosage on the basis of small changes in the results of clotting tests is valueless. Other reviewers have reached different conclusions from the same data and believe that haemorrhage and recurrence can be predicted from clotting test results. ${ }^{19}$ A prudent approach is to check results of clotting tests occasionally to ensure that gross over or under anticoagulation is not occurring, to do these tests more frequently if recurrence or haemorrhage occur, and to use continuous infusion if the risk of haemorrhage is high. The correct dosage is arbitrary but most studies suggest that it should be from 480 to 600 units $/ \mathrm{kg} /$ per day. ${ }^{17}$ During intermittent treatment a slight residual anticoagulant effect should be present before the next dose; this level of anticoagulation is effective ${ }^{17}$ and avoids the high dosage and increased risk of bleeding inherent in giving enough heparin to produce during these troughs of activity the anticoagulant levels that can be achieved continuously by infusion. During continuous infusion the timing of clotting tests should be standardised because of the diurnal variation in heparin activity. ${ }^{18}$ Since the relationship between these variations and the risk of further embolism or haemorrhage is unknown, an arbitrary time (usually the morning) is chosen; but if bleeding becomes a problem further checks when heparin activity is likely to be highest (midnight) may be helpful.

Finally, for how long should heparin treatment be continued? The answer is completely unknown and local practice varies widely from the three or four days needed to establish oral anticoagulation to 10-15 days. ${ }^{17}$ Animal experiments suggest that clots take 7-10 days to become firmly adherent to vessel walls and therefore unlikely to embolise. An arbitrary guideline derived from this finding and from clinical anecdote suggests that heparin should be continued for at least a week after a major thromboembolic episode (deep vein thrombosis or pulmonary embolus) and certainly until the patient is ambulant.

\section{Should venography with a view to venous interrup- tion be considered routinely in patients who have suffered a definite pulmonary embolus?}

The only justification for such an approach would be evidence that it reduces the risk of subsequent emboli. Once a patient has experienced pulmonary embolism a reasonable assumption is that there will be a residual clot in the venous system. This can be shown in $80-90 \%$ of patients either at necropsy ${ }^{20}$ or by venography. This clot represents potential further emboli. The routine use of venous interruption to trap such clot is logical only if there is a lower mortality rate in such patients than in similar patients who receive heparin followed by oral anticoagulant treatment without undergoing venous interruption. Otherwise the routine or even frequent use of venography is not justified since in these circumstances its only use is to guide a further intervention. As usual the relevant data are not available; this comparison has never been made. Although many hundreds of cases of venous interruption with various operations and devices have been reported, ${ }^{19}$ there has been little or no attempt to examine its value critically by prospective study. 
Many series include mixtures of patients, some of whom might be considered to be at very high risk, since they had undergone the procedure after other measures to prevent further embolisation had failed, while others have been treated simply as a prophylactic measure, having had no preceding embolus or even no thromboembolic disease at all. ${ }^{1921}$ The timing of intervention and length of follow up are also very variable.

Since there is no convincing evidence to guide the clinician, he has to remember that standard regimens of anticoagulation are generally easy to administer and produce excellent results. The overall early mortality rate in treated acute pulmonary embolism from further embolism or haemorrhage is $3-5 \%{ }^{7}{ }^{17}$ and deaths due to recurring embolism after hospital discharge are extremely rare. ${ }^{4}$ The results of the routine use of a surgical procedure with a high operative mortality rate $(15 \%$ for ligation or plication of the inferior vena cava, $7 \%$ for the surgical insertion of clips to narrow and compartmentalise the lumen of the vena cava inferior ${ }^{19}$ ) and a high incidence of unpleasant lower limb oedema and venous thrombosis in the later stages $(40 \%)$ can never rival those figures.

The pervenous placement of filters, umbrellas, and so on in the inferior vena cava is safer than surgical ligation or plication and creates less by way of venous complications, but has problems of its own. ${ }^{19}$ Insertion needs experience and difficulties occur in up to $15 \%$ of cases. ${ }^{22}$ The filters may migrate or may themselves embolise and are sometimes sited in the wrong place (14\%). A few patients (3\%) have emboli after placement. The operative mortality of the procedure is probably $1-3 \% .^{19}$

Many authorities on the subject now admit that anticoagulation alone is adequate treatment for nearly all patients ${ }^{2122}$ and that venous interruption, preferably using a filter device (the Greenfield filter seems the easiest to use), should be reserved for patients with either definite recurrence of pulmonary embolism despite adequate anticoagulation or a high risk of emboli combined with a strong contraindication to anticoagulants. Once such a view has been adopted routine venography becomes illogical unless one of these indications for venous interruption is present. Since such patients are rare, amounting possibly to $2 \%$ of all patients experiencing acute pulmonary embolism, ${ }^{1922}$ venous interruption should be carried out only in specialist centres where clinicians have the chance to build up the necessary experience to keep the risks of insertion low.

\section{Conclusions}

Thrombolytic treatment probably benefits patients who suffer a large acute pulmonary embolus but it should not be used unless the embolus causes considerable haemodynamic disturbance. In nearly all other situations thrombolytic treatment is more expensive, more dangerous, but no more effective than heparin. When heparin is given there seems to be little to choose between intermittent injection and continuous infusion. The value of coagulation tests as a guide to adjustment of heparin dosage is not proved and seems to need reassessment in the light of the recent discovery of large diurnal variations in heparin activity. Finally venography, in the setting of a proved pulmonary embolus, is unnecessary unless recurrence of embolism despite adequate anticoagulation or a strong contraindication to anticoagulants makes a venous interruption procedure necessary.

ROGER HAL.L.

Royal Victoria Infurmary and Freeman Hospital Newcastle upon Tyne

\section{References}

1 Barrit DW, Jordon SC. Anticoagulant drugs in the treatment of pulmonary embolism: a controlled trial. Lancet 1960; ; 1309-12.

2 National Co-operative Study. Urokinase pulmonary embolism trial. Circulation 1973;47-48, suppl II: 1108.

3 Miller GAH, Sutton GC, Kerr IH, Gibson RV, Honey $M$. Comparison of streptokinase and heparin in the treatment of isolated acute massive pulmonary embolism. $\mathrm{Br}$ Med J 1971;ii:681-4.

4 Hall RJC, Sutton GC, Kerr IH. Long term prognosis of treated acute massive pulmonary embolism. Br Heart J 1977;39:1128-34.

5 Sharma GVRK, Burleson VA, Sasahara AA. Effect of thrombolytic therapy on capillary blood volume in patients with pulmonary embolism. $N$ Engl J Med 1980;303:842-5.

6 Turnier E, Hill JD, Kerth WJ, Gerbode F. Massive pulmonary embolism. Am J Cardiol 1973;125:611-22.

7 Tibbut DA, Davies JA, Anderson JA, et al. Comparison by controlled clinical trial of streptokinase and heparin in treatment of life-threatening pulmonary embolism. Br Med J 1974;i:343-7.

8 Miller GAH, Hall RJC, Panneth M. Pulmonary embolectomy, heparin and streptokinase-their place in the treatment of acute massive pulmonary embolism. Am Heart J 1977;93:568-74.

9 Stein PD, Willis PW. Diagnosis, prophylaxis and treatment of acute pulmonary embolism. Arch Intern Med 1983;143:991-4.

10 Lemaire F. Pulmonary embolism: what is known, what remains unsolved? Intensive Care Medicine 1984; 10:79-80.

11 Basu D, Gallus A, Hirsh J, Cade J. A prospective study 
of the value of monitoring heparin treatment with the activated partial thromboplastin time. $N$ Engl J Med 1972;287:324-7.

12 Hirsh J, Van Aken WG, Gallus AS, Dollery CT, Cade JF, Yung WL. Heparin kinetics in venous thrombosis and pulmonary embolism. Circulation 1976;53:691-5.

13 Rothschild BM. The role of antithrombin III in the clinical management of pulmonary embolization. $A m \mathbf{J}$ Med 1983;74:529-30.

14 Mant MJ, O'Brien BD, Thong KL, Hammond GW, Birtwhistle RV, Grace MG. Haemorrhagic complications of heparin therapy. Lancet 1977;ii:1133-5.

15 Salzman EW, Deykin D, Shapiro RM, Rosenberg R. Management of heparin therapy. Controlled prospective trial. N Engl J Med 1975;292:1046-50.

16 Glazier RL, Crowell EB. Randomised, prospective trial of continuous v's intermittent heparin therapy. JAMA 1976;263:1365-7.
17 Wilson JE, Bynum LJ, Parkey RW. Heparin therapy in venous thromboembolism. Am J Med 1981;70: 808-16.

18 Decousus HA, Croze M, Levi FA, et al. Circadian changes in anticoagulant effect of heparin infused at constant rate. Br Med J 1985;290:341-4.

19 Bomalski JS, Martin GJ, Hughes RL, Yao JST. Inferior vena cava interruption in the management of pulmonary embolism. Chest 1982;82:767-74.

20 Sevitt S, Gallagher N. Venous thrombosis and pulmonary embolism. A clinico-pathological study in injured and burned patients. Br J Surg 1961;48:475-89.

21 Greenfield LJ. Current indications for and results of Greenfield filter placement. Journal of Vascular Surgery 1984;1:502-4.

22 Brewster DC. Introduction to symposium on transvenous vena cava interruption. Journal of Vascular Surgery 1984;1:487-90. 